\title{
A system of conjugate functions on parabolic Bloch spaces
}

\author{
By Yôsuke Hishikawa, Masaharu Nishio and Masahiro YAmadA
}

(Received Apr. 12, 2016)

(Revised Jan. 10, 2017)

\begin{abstract}
The parabolic Bloch space is the set of all solutions $u$ of the parabolic operator $L^{(\alpha)}$ with the finite Bloch norm $\|u\|_{\mathcal{B}_{\alpha}(\sigma)}$. In this paper, we introduce $L^{(\alpha)}$-conjugates of parabolic Bloch functions, and investigate several properties. As an application, we give an isomorphism theorem on parabolic Bloch spaces.
\end{abstract}

\section{Introduction.}

Let $H$ be the upper half-space of the $(n+1)$-dimensional Euclidean space $\mathbb{R}^{n+1}(n \geq$ $1)$, that is, $H=\left\{(x, t) \in \mathbb{R}^{n+1} ; x \in \mathbb{R}^{n}, t>0\right\}$. For $0<\alpha \leq 1$, the parabolic operator $L^{(\alpha)}$ is defined by

$$
L^{(\alpha)}=\partial_{t}+\left(-\Delta_{x}\right)^{\alpha},
$$

where $\partial_{t}=\partial / \partial t$ and $\Delta_{x}$ is the Laplacian with respect to $x$. Let $C(H)$ be the set of all real-valued continuous functions on $H$, and $C^{k}(H)$ the set of all $k$ times continuously differentiable functions on $H$. A function $u \in C(H)$ is said to be $L^{(\alpha)}$-harmonic if $L^{(\alpha)} u=$ 0 in the sense of distributions (for details, see Section 2). Put $m(\alpha)=\min \{1,1 / 2 \alpha\}$. Let $\sigma>-m(\alpha)$ be a real number. We denote by $\mathcal{B}_{\alpha}(\sigma)$ the set of all $L^{(\alpha)}$-harmonic functions $u \in C^{1}(H)$ which satisfy

$$
\|u\|_{\mathcal{B}_{\alpha}(\sigma)}:=\sup _{(x, t) \in H} t^{\sigma}\left\{t^{1 / 2 \alpha}\left|\nabla_{x} u(x, t)\right|+t\left|\partial_{t} u(x, t)\right|\right\}<\infty
$$

where $\nabla_{x}=\left(\partial_{1}, \ldots, \partial_{n}\right)$ and $\partial_{j}=\partial / \partial x_{j}$. We also denote by $\widetilde{\mathcal{B}}_{\alpha}(\sigma)$ the set of all functions $u \in \mathcal{B}_{\alpha}(\sigma)$ which satisfy $u(0,1)=0$. We call $\widetilde{\mathcal{B}}_{\alpha}(\sigma)$ (or $\mathcal{B}_{\alpha}(\sigma)$ ) the parabolic Bloch space. We remark that $\widetilde{\mathcal{B}}_{\alpha}(\sigma)$ is a Banach space with the norm $\|\cdot\|_{\mathcal{B}_{\alpha}(\sigma)}$ (see $\left.[\mathbf{6}]\right)$. It is well known that the fundamental solutions of $L^{(1 / 2)}$ and $L^{(1)}$ are the Poisson and heat kernels, respectively. In other cases $(\alpha \neq 1 / 2,1)$, simple explicit expressions for the fundamental solutions are not known. Furthermore, we also note that $\widetilde{\mathcal{B}}_{1 / 2}(0)$ coincides with the harmonic Bloch space of Ramey and Yi [13].

The fractional Laplacian $(-\Delta)^{\alpha}$ is actively investigated in the theory of partial differential equations. Recently, there are various studies which are concerned with generalized

2010 Mathematics Subject Classification. Primary 35K05; Secondary 42A50, 26A33.

Key Words and Phrases. parabolic operator of fractional order, heat equation, Bloch space, conjugate function.

This work was supported in part by Grant-in-Aid for Scientific Research (C) No.16K05198 and No.15K04934, Japan Society for the Promotion of Science. 
heat equations defined by the fractional Laplacian or fractional differential operators (for example, see [1], [8], and [9]). On the other hand, Nishio, Shimomura, and Suzuki [11] introduced new function spaces which consist of $L^{(\alpha)}$-harmonic functions, and established fundamental properties of those spaces using potential theory method in [7]. The background of our investigation is based on their theory in [11].

In this paper, we study a notion of conjugate functions on parabolic Bloch spaces for all $0<\alpha \leq 1$, and give several properties which are parallel to theory of the classical harmonic conjugates of Stein and Weiss [14]. Stein and Weiss introduced harmonic conjugates for harmonic functions on $H$. According to [14], harmonic conjugates are defined by the generalized Cauchy-Riemann equations. Recently, we study $\alpha$-parabolic conjugate functions on parabolic Bloch spaces in [4]. The study of [4] is an extension of harmonic conjugates of [14]. However, our extension of [4] is not satisfactory, because there exist parabolic Bloch functions which do not have $\alpha$-parabolic conjugate functions. Therefore, in this paper, we introduce the notion of $L^{(\alpha)}$-conjugates to parabolic Bloch spaces, and thus obtain completely satisfying results which are parallel to theory of the harmonic conjugates. Moreover, in Section 5 of this paper, we introduce a notion of $(\kappa, \nu)$-conjugates which is a full generalization of our conjugacy. Actually, in Theorem 5.4 , we show that if $\kappa=1$ and $\nu=(1 / \alpha)-1$ then $(\kappa, \nu)$-conjugacy coincides with $\alpha$ parabolic conjugacy in Theorem A below; if $\kappa=\nu=1 / 2 \alpha$ then $(\kappa, \nu)$-conjugacy coincides with $L^{(\alpha)}$-conjugacy in Theorem 1 below.

To state our results, we give some notations. For a real number $\kappa$, let $\mathcal{D}_{t}^{\kappa}=\left(-\partial_{t}\right)^{\kappa}$ be a fractional differential operator, and $\mathcal{F} \mathcal{C}^{\kappa}$ the class of functions $\varphi$ on $\mathbb{R}_{+}=(0, \infty)$ such that $\mathcal{D}_{t}^{\kappa} \varphi$ is well-defined (for the explicit definitions of $\mathcal{D}_{t}^{\kappa}$ and $\mathcal{F} \mathcal{C}^{\kappa}$, see Section 2). First, we present the definition of harmonic conjugates for harmonic functions on $H$. According to [14], harmonic conjugates are defined by the generalized Cauchy-Riemann equations.

Definition A $([\mathbf{1 4}])$. Let $u$ be a function on $H$. An $n$-tuple $\left(v_{1}, \ldots, v_{n}\right)$ is called a harmonic conjugate of $u$ if an $(n+1)$-tuple $\left(v_{1}, \ldots, v_{n}, u\right)$ satisfies the following generalized Cauchy-Riemann equations;

$$
\begin{aligned}
& \partial_{j} v_{k}=\partial_{k} v_{j}(1 \leq j, k \leq n), \\
& \partial_{j} u=-\mathcal{D}_{t} v_{j}(1 \leq j \leq n), \\
& \mathcal{D}_{t} u=\sum_{j=1}^{n} \partial_{j} v_{j} .
\end{aligned}
$$

Next, we describe our previous results. In [4], we extended the notion of harmonic conjugates by fractional differential operators. Our previous extension in [4] is as follows.

Definition B (Definitions 1 and 2 of [4]). Let $0<\alpha \leq 1$ and let $u$ be a function on $H$. An $n$-tuple $\left(v_{1}, \ldots, v_{n}\right)$ is called an $\alpha$-parabolic conjugate function of $u$ if $v_{j} \in C^{1}(H)$, $u(x, \cdot) \in \mathcal{F C}^{(1 / \alpha)-1}$, and an $(n+1)$-tuple $\left(v_{1}, \ldots, v_{n}, u\right)$ satisfies the following equations;

$$
\begin{gathered}
\partial_{j} v_{k}=\partial_{k} v_{j}(1 \leq j, k \leq n), \\
\partial_{j} u=-\mathcal{D}_{t} v_{j}(1 \leq j \leq n),
\end{gathered}
$$


and

$$
\sum_{j=1}^{n} \partial_{j} v_{j}= \begin{cases}\mathcal{D}_{t}^{(1 / \alpha)-1} u & (0<\alpha<1), \\ u-\lim _{t \rightarrow \infty} u(0, t) & (\alpha=1) .\end{cases}
$$

We remark that (1/2)-parabolic conjugate functions coincide with harmonic conjugates. The following theorem is the main result of $[\mathbf{4}]$.

Theorem A (Theorems 1 and 2 of [4]). Let $0<\alpha \leq 1, \sigma>-m(\alpha)$, and $u \in$ $\widetilde{\mathcal{B}}_{\alpha}(\sigma)$. Put $\eta:=(1 / 2 \alpha)-1+\sigma$. If $\eta>-1 / 2 \alpha$, then there exists a unique $\alpha$-parabolic conjugate function $\left(v_{1}, \ldots, v_{n}\right)$ of $u$ such that $v_{j} \in \widetilde{\mathcal{B}}_{\alpha}(\eta)$. Also, there exists a constant $C>0$ independent of $u$ such that

$$
C^{-1}\|u\|_{\mathcal{B}_{\alpha}(\sigma)} \leq \sum_{j=1}^{n}\left\|v_{j}\right\|_{\mathcal{B}_{\alpha}(\eta)} \leq C\|u\|_{\mathcal{B}_{\alpha}(\sigma)} .
$$

We give a remark of Theorem A. When $0<\alpha \leq 1 / 2$, the condition of $\eta$ is always satisfied. However, when $1 / 2<\alpha \leq 1$, there exists $\sigma>-m(\alpha)$ such that $\eta \leq-1 / 2 \alpha$. In other words, there exists $u \in \widetilde{\mathcal{B}}_{\alpha}(\sigma)$ which does not have an $\alpha$-parabolic conjugate function. In order to solve this problem, we need to introduce the definition of $L^{(\alpha)}$. conjugates. Now, we describe the main results of this paper.

Definition 1. Let $0<\alpha \leq 1$ and let $u$ be a function on $H$. An $n$-tuple $\left(v_{1}, \ldots, v_{n}\right)$ is called an $L^{(\alpha)}$-conjugate of $u$ if $v_{j}, u \in C^{1}(H), v_{j}(x, \cdot), u(x, \cdot) \in \mathcal{F C}^{1 / 2 \alpha}$, and an $(n+1)$-tuple $\left(v_{1}, \ldots, v_{n}, u\right)$ satisfies the following equations;

$$
\begin{aligned}
& \partial_{j} v_{k}=\partial_{k} v_{j}(1 \leq j, k \leq n), \\
& \partial_{j} u=-\mathcal{D}_{t}^{1 / 2 \alpha} v_{j}(1 \leq j \leq n),
\end{aligned}
$$

and

$$
\mathcal{D}_{t}^{1 / 2 \alpha} u=\sum_{j=1}^{n} \partial_{j} v_{j}
$$

We remark that $L^{(1 / 2)}$-conjugates coincide with harmonic conjugates. Thus, Definition 1 is also an extension of Definition A. Theorem 1 is the main theorem of this paper.

TheOREM 1. Let $0<\alpha \leq 1, \sigma>-m(\alpha)$, and $u \in \widetilde{\mathcal{B}}_{\alpha}(\sigma)$. Then, there exists a unique $L^{(\alpha)}$-conjugate $\left(v_{1}, \ldots, v_{n}\right)$ of $u$ such that $v_{j} \in \widetilde{\mathcal{B}}_{\alpha}(\sigma)$. Moreover, there exists a constant $C>0$ independent of $u$ such that

$$
C^{-1}\|u\|_{\mathcal{B}_{\alpha}(\sigma)} \leq \sum_{j=1}^{n}\left\|v_{j}\right\|_{\mathcal{B}_{\alpha}(\sigma)} \leq C\|u\|_{\mathcal{B}_{\alpha}(\sigma)}
$$

Theorem 1 asserts that the $L^{(\alpha)}$-conjugate of $u \in \widetilde{\mathcal{B}}_{\alpha}(\sigma)$ always exists for all $0<$ 
$\alpha \leq 1$ and $\sigma>-m(\alpha)$. Furthermore, for $u \in \widetilde{\mathcal{B}}_{\alpha}(\sigma)$, the function $v_{j}$ belongs to the same Banach space $\widetilde{\mathcal{B}}_{\alpha}(\sigma)$.

We also give an inversion theorem of Theorem 1 .

Theorem 2. Let $0<\alpha \leq 1, \sigma>-m(\alpha)$. Suppose an $n$-tuple $\left(v_{1}, \ldots, v_{n}\right)$ satisfies $v_{j} \in \widetilde{\mathcal{B}}_{\alpha}(\sigma)$ and (1.1). Then, there exists a unique function $u \in \widetilde{\mathcal{B}}_{\alpha}(\sigma)$ such that $\left(v_{1}, \ldots, v_{n}\right)$ is the $L^{(\alpha)}$-conjugate of $u$. Moreover, there exists a constant $C>0$ independent of $v_{j}$ such that

$$
C^{-1} \sum_{j=1}^{n}\left\|v_{j}\right\|_{\mathcal{B}_{\alpha}(\sigma)} \leq\|u\|_{\mathcal{B}_{\alpha}(\sigma)} \leq C \sum_{j=1}^{n}\left\|v_{j}\right\|_{\mathcal{B}_{\alpha}(\sigma)} .
$$

As an application, we give an isomorphism theorem on parabolic Bloch spaces. The study of parabolic Bloch spaces is closely related to that of parabolic Bergman spaces. Let $0<\alpha \leq 1,1 \leq p<\infty$, and $\lambda>-1$. We denote by $\boldsymbol{b}_{\alpha}^{p}(\lambda)$ the parabolic Bergman spaces and the norm is denoted by $\|\cdot\|_{L^{p}(\lambda)}$ (for the explicit definition, see Section 6 ). We already gave the following theorem in the previous paper [5], which is an isomorphism theorem on parabolic Bergman spaces.

Theorem B (Theorem 5.4 and Remark 5.5 of [5]). Let $0<\alpha \leq 1,1 \leq p<\infty$, and $\lambda_{1}, \lambda_{2}>-1$. Then, $\boldsymbol{b}_{\alpha}^{p}\left(\lambda_{1}\right) \cong \boldsymbol{b}_{\alpha}^{p}\left(\lambda_{2}\right)$ under the relation $\mathcal{D}_{t}^{-\lambda_{1} / p} u=\mathcal{D}_{t}^{-\lambda_{2} / p} v$ for $u \in \boldsymbol{b}_{\alpha}^{p}\left(\lambda_{1}\right)$ and $v \in \boldsymbol{b}_{\alpha}^{p}\left(\lambda_{2}\right)$. Moreover, there exists a constant $C>0$ such that

$$
C^{-1}\|v\|_{L^{p}\left(\lambda_{2}\right)} \leq\|u\|_{L^{p}\left(\lambda_{1}\right)} \leq C\|v\|_{L^{p}\left(\lambda_{2}\right)} .
$$

The following is an isomorphism theorem on parabolic Bloch spaces.

TheOREM 3. Let $0<\alpha \leq 1$ and $\sigma_{1}, \sigma_{2}>-m(\alpha)$ be real numbers. Then, $\widetilde{\mathcal{B}}_{\alpha}\left(\sigma_{1}\right) \cong$ $\widetilde{\mathcal{B}}_{\alpha}\left(\sigma_{2}\right)$ under the relation $\mathcal{D}_{t}^{-\sigma_{1}+\kappa} u=\mathcal{D}_{t}^{-\sigma_{2}+\kappa} v$ for $u \in \widetilde{\mathcal{B}}_{\alpha}\left(\sigma_{1}\right)$ and $v \in \widetilde{\mathcal{B}}_{\alpha}\left(\sigma_{2}\right)$, where $\kappa$ is a real number such that $\kappa>\max \left\{0, \sigma_{1}, \sigma_{2}\right\}$. Moreover, there exists a constant $C>0$ such that

$$
C^{-1}\|v\|_{\mathcal{B}_{\alpha}\left(\sigma_{2}\right)} \leq\|u\|_{\mathcal{B}_{\alpha}\left(\sigma_{1}\right)} \leq C\|v\|_{\mathcal{B}_{\alpha}\left(\sigma_{2}\right)} .
$$

Finally, we describe the construction of this paper. In Section 2, we introduce $L^{(\alpha)}$-harmonic functions and fractional differential operators, and present their basic properties. In Section 3, we give basic properties of parabolic Bloch functions. In Section 4, we give the proof of Theorems 1 and 2. In Section 5, we extend the notion of $L^{(\alpha)}$-conjugates called $(\kappa, \nu)$-conjugates, and give their properties. In Section 6 , we give the proof of Theorem 3, which is an application of Sections 4 and 5 .

\section{Preliminaries.}

In this section, we describe definitions of $L^{(\alpha)}$-harmonic functions and fractional differential operators, and present basic properties of fractional calculus of $L^{(\alpha)}$-harmonic functions. We recall the definition of $L^{(\alpha)}$-harmonic functions. We describe the operator $\left(-\Delta_{x}\right)^{\alpha}$. Since the case $\alpha=1$ is trivial, we only describe the case $0<\alpha<1$. Let 
$d V(x, t)=d x d t$ be the Lebesgue volume measure on $H$. Let $C^{\infty}(H)$ be the set of all infinitely differentiable functions on $H$ and $C_{c}^{\infty}(H)$ the set of all functions in $C^{\infty}(H)$ with compact support. For $0<\alpha<1,\left(-\Delta_{x}\right)^{\alpha}$ is the convolution operator defined by

$$
\left(-\Delta_{x}\right)^{\alpha} \psi(x, t)=-c_{n, \alpha} \lim _{\delta \rightarrow 0^{+}} \int_{|y-x|>\delta}(\psi(y, t)-\psi(x, t))|y-x|^{-n-2 \alpha} d y
$$

for all $\psi \in C_{c}^{\infty}(H)$ and $(x, t) \in H$, where $c_{n, \alpha}=-4^{\alpha} \pi^{-n / 2} \Gamma((n+2 \alpha) / 2) / \Gamma(-\alpha)>0$. A function $u \in C(H)$ is said to be $L^{(\alpha)}$-harmonic on $H$ if $u$ satisfies the following condition: for every $\psi \in C_{c}^{\infty}(H)$,

$$
\int_{H}\left|u \cdot \widetilde{L}^{(\alpha)} \psi\right| d V<\infty \quad \text { and } \quad \int_{H} u \cdot \widetilde{L}^{(\alpha)} \psi d V=0
$$

where $\widetilde{L}^{(\alpha)}=-\partial_{t}+\left(-\Delta_{x}\right)^{\alpha}$ is the adjoint operator of $L^{(\alpha)}$. By (2.1) and the compactness of $\operatorname{supp}(\psi)$ (the support of $\psi$ ), there exist $0<t_{1}<t_{2}<\infty$ and a constant $C>0$ such that $\operatorname{supp}\left(\widetilde{L}^{(\alpha)} \psi\right) \subset S=\mathbb{R}^{n} \times\left[t_{1}, t_{2}\right]$ and $\left|\widetilde{L}^{(\alpha)} \psi(x, t)\right| \leq C(1+|x|)^{-n-2 \alpha}$ for all $(x, t) \in S$. Thus, the integrability condition of $(2.2)$ is equivalent to the following: for any $0<t_{1}<t_{2}<\infty$,

$$
\int_{t_{1}}^{t_{2}} \int_{\mathbb{R}^{n}}|u(x, t)|(1+|x|)^{-n-2 \alpha} d V(x, t)<\infty .
$$

We introduce the fundamental solution of $L^{(\alpha)}$. For $x \in \mathbb{R}^{n}$, the fundamental solution $W^{(\alpha)}$ of $L^{(\alpha)}$ is defined by

$$
W^{(\alpha)}(x, t)= \begin{cases}\frac{1}{(2 \pi)^{n}} \int_{\mathbb{R}^{n}} \exp \left(-t|\xi|^{2 \alpha}+\sqrt{-1} x \cdot \xi\right) d \xi & t>0 \\ 0 & t \leq 0\end{cases}
$$

where $x \cdot \xi$ denotes the inner product on $\mathbb{R}^{n}$. It is known that $W^{(\alpha)}$ is $L^{(\alpha)}$-harmonic on $H$ and $W^{(\alpha)} \in C^{\infty}(H)$.

We present definitions of fractional integral and differential operators. Let $C\left(\mathbb{R}_{+}\right)$ be the set of all continuous functions on $\mathbb{R}_{+}=(0, \infty)$. For a positive real number $\kappa$, let $\mathcal{F C}^{-\kappa}$ be the set of all functions $\varphi \in C\left(\mathbb{R}_{+}\right)$such that there exists a constant $\varepsilon>0$ with $\varphi(t)=O\left(t^{-\kappa-\varepsilon}\right)$ as $t \rightarrow \infty$. We remark that $\mathcal{F} \mathcal{C}^{-\nu} \subset \mathcal{F} \mathcal{C}^{-\kappa}$ if $0<\kappa \leq \nu$. For $\varphi \in \mathcal{F} \mathcal{C}^{-\kappa}$, we can define the fractional integral of $\varphi$ with order $\kappa$ by

$$
\mathcal{D}_{t}^{-\kappa} \varphi(t)=\frac{1}{\Gamma(\kappa)} \int_{0}^{\infty} \tau^{\kappa-1} \varphi(t+\tau) d \tau, \quad t \in \mathbb{R}_{+} .
$$

Furthermore, let $\mathcal{F C} \mathcal{C}^{\kappa}$ be the set of all functions $\varphi \in C\left(\mathbb{R}_{+}\right)$such that $\partial_{t}^{\lceil\kappa\rceil} \varphi \in$ $\mathcal{F C}^{-(\lceil\kappa\rceil-\kappa)}$, where $\lceil\kappa\rceil$ is the smallest integer greater than or equal to $\kappa$. In particular, we will write $\mathcal{F} \mathcal{C}^{0}=C\left(\mathbb{R}_{+}\right)$. For $\varphi \in \mathcal{F} \mathcal{C}^{\kappa}$, we can also define the fractional derivative of $\varphi$ with order $\kappa$ by

$$
\mathcal{D}_{t}^{\kappa} \varphi(t)=\mathcal{D}_{t}^{-(\lceil\kappa\rceil-\kappa)}\left(-\partial_{t}\right)^{\lceil\kappa\rceil} \varphi(t), \quad t \in \mathbb{R}_{+} .
$$


Also, we define $\mathcal{D}_{t}^{0} \varphi=\varphi$. We may often call both (2.4) and (2.5) the fractional derivative of $\varphi$ with order $\kappa$. Moreover, we call $\mathcal{D}_{t}^{\kappa}$ the fractional differential operator with order $\kappa$.

We describe basic properties of fractional derivatives of the fundamental solution $W^{(\alpha)}$, which are given in [2]. Let $\mathbb{N}_{0}=\mathbb{N} \cup\{0\}$. For a multi-index $\beta=\left(\beta_{1}, \ldots, \beta_{n}\right) \in \mathbb{N}_{0}^{n}$, let $\partial_{x}^{\beta}=\partial^{|\beta|} / \partial x_{1} \beta_{1} \cdots \partial x_{n}{ }^{\beta_{n}}$, where $|\beta|=\beta_{1}+\cdots+\beta_{n}$. The following lemma is given by Theorem 3.1 of $[\mathbf{2}]$ and Theorem 4.1 of [3].

Lemma 2.1. Let $0<\alpha \leq 1, \beta \in \mathbb{N}_{0}^{n}$, and let $\kappa>-n / 2 \alpha$ be a real number. Then, the following statements hold.

(1) The both derivatives $\partial_{x}^{\beta} \mathcal{D}_{t}^{\kappa} W^{(\alpha)}(x, t)$ and $\mathcal{D}_{t}^{\kappa} \partial_{x}^{\beta} W^{(\alpha)}(x, t)$ are well-defined and $\partial_{x}^{\beta} \mathcal{D}_{t}^{\kappa} W^{(\alpha)}(x, t)=\mathcal{D}_{t}^{\kappa} \partial_{x}^{\beta} W^{(\alpha)}(x, t)$. Moreover, there exists a constant $C>0$ such that

$$
\left|\partial_{x}^{\beta} \mathcal{D}_{t}^{\kappa} W^{(\alpha)}(x, t)\right| \leq C\left(t+|x|^{2 \alpha}\right)^{-((n+|\beta|) / 2 \alpha)-\kappa}
$$

for all $(x, t) \in H$.

(2) Let $\nu$ be a real number such that $\kappa+\nu>-n / 2 \alpha$. Then,

$$
\mathcal{D}_{t}^{\nu} \partial_{x}^{\beta} \mathcal{D}_{t}^{\kappa} W^{(\alpha)}(x, t)=\partial_{x}^{\beta} \mathcal{D}_{t}^{\kappa+\nu} W^{(\alpha)}(x, t)
$$

for all $(x, t) \in H$.

(3) $\partial_{x}^{\beta} \mathcal{D}_{t}^{\kappa} W^{(\alpha)}$ is $L^{(\alpha)}$-harmonic on $H$.

(4) $\left(\mathcal{D}_{t}^{1 / \alpha}+\Delta_{x}\right) W^{(\alpha)}(x, t)=0$ for all $(x, t) \in H$.

Let $\gamma \in \mathbb{N}_{0}^{n}$ be a multi-index, and $\nu>-n / 2 \alpha$ be a real number. We define a function $\omega_{\alpha}^{\gamma, \nu}$ on $H \times H$ by

$$
\omega_{\alpha}^{\gamma, \nu}(x, t ; y, s):=\partial_{x}^{\gamma} \mathcal{D}_{t}^{\nu} W^{(\alpha)}(x-y, t+s)-\partial_{x}^{\gamma} \mathcal{D}_{t}^{\nu} W^{(\alpha)}(-y, 1+s)
$$

for all $(x, t),(y, s) \in H$. The function $\omega_{\alpha}^{\gamma, \nu}$ plays an important role for the study of parabolic Bloch spaces. Basic properties of the function $\omega_{\alpha}^{\gamma, \nu}$ are given by Lemma 5.6 of $[6]$.

Lemma 2.2. Let $0<\alpha \leq 1, \sigma>-m(\alpha), \gamma \in \mathbb{N}_{0}^{n}$, and $\nu>-n / 2 \alpha$. Then, the following statements hold.

(1) For every $(x, t) \in H$, there exists a constant $C=C(n, \alpha, \gamma, \nu, x, t)>0$ such that

$$
\left|\omega_{\alpha}^{\gamma, \nu}(x, t ; y, s)\right| \leq C\left(1+s+|y|^{2 \alpha}\right)^{-((n+|\gamma|) / 2 \alpha)-\nu-m(\alpha)}
$$

for all $(y, s) \in H$.

(2) If $\rho>-1$ and $\eta:=(|\gamma| / 2 \alpha)+\nu-\rho-1>-m(\alpha)$, then there exists a constant $C=C(n, \alpha, \gamma, \nu, \rho)>0$ such that

$$
\int_{H}\left|\omega_{\alpha}^{\gamma, \nu}(x, t ; y, s)\right| s^{\rho} d V(y, s) \leq C F_{\alpha, \eta}(x, t)
$$


for all $(x, t) \in H$, where

$$
F_{\alpha, \eta}:= \begin{cases}1+|x|^{-2 \alpha \eta}+t^{-\eta} & (0>\eta>-m(\alpha)) \\ 1+\log (1+|x|)+|\log t| & (\eta=0) \\ 1+t^{-\eta} & (\eta>0) .\end{cases}
$$

Finally, we present the following lemma.

Lemma 2.3 (Lemma 5 of $[\mathbf{1 2}]$ ). Let $\theta, c \in \mathbb{R}$. If $\theta>-1$ and $\theta-c+(n / 2 \alpha)+1<0$, then there exists a constant $C=C(n, \alpha, \theta, c)>0$ such that

$$
\int_{H} \frac{s^{\theta}}{\left(t+s+|x-y|^{2 \alpha}\right)^{c}} d V(y, s)=C t^{\theta-c+(n / 2 \alpha)+1}
$$

for all $(x, t) \in H$.

\section{Basic properties of parabolic Bloch functions.}

In this section, we give basic properties of parabolic Bloch functions. First, we present basic properties of fractional calculus on parabolic Bloch spaces. The following lemma is given by Proposition 5.4 of [6] and Lemma 6.1 of [4].

LEMma 3.1. Let $0<\alpha \leq 1, \sigma>-m(\alpha), \gamma \in \mathbb{N}_{0}^{n}$ be a multi-index, and $\kappa$ be a real number such that $\kappa=0$ or $\kappa>\max \{0,-\sigma\}$. If $u \in \mathcal{B}_{\alpha}(\sigma)$, then the following statements hold.

(1) The derivatives $\partial_{x}^{\gamma} \mathcal{D}_{t}^{\kappa} u(x, t)$ and $\mathcal{D}_{t}^{\kappa} \partial_{x}^{\gamma} u(x, t)$ can be defined, and the equation $\partial_{x}^{\gamma} \mathcal{D}_{t}^{\kappa} u(x, t)=\mathcal{D}_{t}^{\kappa} \partial_{x}^{\gamma} u(x, t)$ holds. Furthermore, if $(\gamma, \kappa) \neq(0,0)$, then there exists a constant $C=C(n, \alpha, \sigma, \gamma, \kappa)>0$ such that

$$
\left|\partial_{x}^{\gamma} \mathcal{D}_{t}^{\kappa} u(x, t)\right| \leq C t^{-((|\gamma| / 2 \alpha)+\kappa+\sigma)}\|u\|_{\mathcal{B}_{\alpha}(\sigma)}
$$

for all $(x, t) \in H$.

(2) If $\nu=0$ or $\nu>\max \{0,-\sigma\}$, then

$$
\mathcal{D}_{t}^{\nu} \partial_{x}^{\gamma} \mathcal{D}_{t}^{\kappa} u(x, t)=\partial_{x}^{\gamma} \mathcal{D}_{t}^{\nu+\kappa} u(x, t) .
$$

Furthermore, if $\nu<0$, then (3.1) also holds whenever $\nu<\sigma$ and $\nu+\kappa>$ $\max \{0,-\sigma\}$.

(3) $\partial_{x}^{\gamma} \mathcal{D}_{t}^{\kappa} u$ is $L^{(\alpha)}$-harmonic on $H$.

(4) $\left(\mathcal{D}_{t}^{1 / \alpha}+\Delta_{x}\right) u(x, t)=0$ for all $(x, t) \in H$.

The following lemma is the reproducing formula on parabolic Bloch spaces.

Lemma 3.2 (Theorem 5.7 of $[\mathbf{6}]$ ). Let $0<\alpha \leq 1$ and $\sigma>-m(\alpha)$. If real numbers $\kappa \in \mathbb{R}_{+}$and $\nu \in \mathbb{R}$ satisfy $\kappa>-\sigma$ and $\nu>\sigma$, then 


$$
u(x, t)-u(0,1)=\frac{2^{\kappa+\nu}}{\Gamma(\kappa+\nu)} \int_{H} \mathcal{D}_{t}^{\kappa} u(y, s) \omega_{\alpha}^{0, \nu}(x, t ; y, s) s^{\kappa+\nu-1} d V(y, s)
$$

for all $u \in \mathcal{B}_{\alpha}(\sigma)$ and $(x, t) \in H$. If $\kappa=0$ and $\nu>\max \{0, \sigma\}$, then (3.2) also holds.

Next, we introduce the following integral operator induced by $\omega_{\alpha}^{\kappa, \nu}$. Let $0<\alpha \leq 1$. Let $\gamma \in \mathbb{N}_{0}^{n}$ be a multi-index, and let $\nu, \rho$ be real numbers. For a function $f$ on $H$, the integral operator $\Pi_{\alpha}^{\gamma, \nu, \rho}$ is defined by

$$
\Pi_{\alpha}^{\gamma, \nu, \rho} f(x, t):=\int_{H} f(y, s) \omega_{\alpha}^{\gamma, \nu}(x, t ; y, s) s^{\rho} d V(y, s) .
$$

Some of the basic properties of the integral operators $\Pi_{\alpha}^{\gamma, \nu, \rho}$ are as follows. We denote by $L^{\infty}(H)$ the set of all Lebesgue measurable functions $f$ on $H$ which satisfy

$$
\|f\|_{L^{\infty}}:=\operatorname{esssup}\{|f(x)|:(x, t) \in H\}<\infty .
$$

Lemma 3.3. Let $0<\alpha \leq 1, \gamma \in \mathbb{N}_{0}^{n}, \nu>-n / 2 \alpha$, and $\rho>-1$. Put $\eta:=$ $(|\gamma| / 2 \alpha)+\nu-\rho-1$. If $\eta>-m(\alpha)$, then the following statements hold:

(1) $\Pi_{\alpha}^{\gamma, \nu, \rho}$ is a bounded operator from $L^{\infty}(H)$ to $\widetilde{\mathcal{B}}_{\alpha}(\eta)$.

(2) Let $\kappa$ be a real number such that $\kappa>\max \{0,-\eta\}$. Then,

$$
\mathcal{D}_{t}^{\kappa} \Pi_{\alpha}^{\gamma, \nu, \rho} f(x, t)=\int_{H} f(y, s) \partial_{x}^{\gamma} \mathcal{D}_{t}^{\kappa+\nu} W^{(\alpha)}(x-y, t+s) s^{\rho} d V(y, s)
$$

holds for all $f \in L^{\infty}(H)$ and $(x, t) \in H$.

(3) Let $\beta \in \mathbb{N}_{0}^{n}$ with $|\beta| \neq 0$. Moreover, let $\kappa$ be a real number such that $\kappa>-(|\beta| / 2 \alpha)-$ $\eta$ and $\kappa+\nu>-n / 2 \alpha$. Then,

$$
\mathcal{D}_{t}^{\kappa} \partial_{x}^{\beta} \Pi_{\alpha}^{\gamma, \nu, \rho} f(x, t)=\int_{H} f(y, s) \partial_{x}^{\beta+\gamma} \mathcal{D}_{t}^{\kappa+\nu} W^{(\alpha)}(x-y, t+s) s^{\rho} d V(y, s)
$$

holds for all $f \in L^{\infty}(H)$ and $(x, t) \in H$.

\section{Proof.}

(1) The essential proof is already given by Proposition 4.1 of [4].

(2) Let $\kappa>\max \{0,-\eta\}$. Since $\kappa>0$, the differentiation under the integral sign shows that

$$
\begin{aligned}
& \mathcal{D}_{t}^{\kappa} \Pi_{\alpha}^{\gamma, \nu, \rho} f(x, t) \\
& =\frac{1}{\Gamma(\lceil\kappa\rceil-\kappa)} \int_{0}^{\infty} \tau^{\lceil\kappa\rceil-\kappa-1} \int_{H} f(y, s) \partial_{x}^{\gamma} \mathcal{D}_{t}^{\lceil\kappa\rceil+\nu} W^{(\alpha)}(x-y, t+\tau+s) s^{\rho} d V(y, s) d \tau .
\end{aligned}
$$

Here, (1) of Lemma 2.1 and Lemma 2.3 imply that 


$$
\int_{0}^{\infty} \int_{H} \tau^{\lceil\kappa\rceil-\kappa-1}\left|f(y, s) \partial_{x}^{\gamma} \mathcal{D}_{t}^{\lceil\kappa\rceil+\nu} W^{(\alpha)}(x-y, t+\tau+s)\right| s^{\rho} d V(y, s) d \tau<\infty,
$$

because $\kappa>-\eta$. Therefore, we can get (3.4) by the Fubini theorem and (2) of Lemma 2.1.

(3) The proof is similar to that of (2). Thus, we omit the proof.

Finally, we present estimates of normal derivative norms of parabolic Bloch functions.

Lemma 3.4 (Theorem 5.9 of $[6]$ ). Let $0<\alpha \leq 1$ and $\sigma>-m(\alpha)$. Then, for every real number $\kappa>\max \{0,-\sigma\}$, there exists a constant $C=C(n, \alpha, \sigma, \kappa)>0$ independent of $u$ such that

$$
C^{-1}\|u\|_{\mathcal{B}_{\alpha}(\sigma)} \leq\left\|t^{\kappa+\sigma} \mathcal{D}_{t}^{\kappa} u\right\|_{L^{\infty}} \leq C\|u\|_{\mathcal{B}_{\alpha}(\sigma)}
$$

for all $u \in \widetilde{\mathcal{B}}_{\alpha}(\sigma)$.

\section{4. $L^{(\alpha)}$-conjugates on parabolic Bloch spaces.}

In this section, we give the main results of this paper. First, we give the proof of Theorem 1.

Proof of Theorem 1. Let $u \in \widetilde{\mathcal{B}}_{\alpha}(\sigma)$. For an integer $1 \leq j \leq n$, we define a function $v_{j}$ on $H$ by

$$
v_{j}(x, t)=-\frac{2^{\sigma+2}}{\Gamma(\sigma+2)} \Pi_{\alpha}^{\gamma(j), \sigma+1-(1 / 2 \alpha), 0}\left(s^{\sigma+1} \mathcal{D}_{t} u\right)(x, t),
$$

where $\gamma(j)=\left(\delta_{j 1}, \ldots, \delta_{j n}\right) \in \mathbb{N}_{0}^{n}$ and $\delta_{j \ell}$ is the Kronecker $\delta$. Since $s^{\sigma+1} \mathcal{D}_{t} u \in L^{\infty}(H)$, (1) of Lemma 3.3 implies that $v_{j} \in \widetilde{\mathcal{B}}_{\alpha}(\sigma)$ and there exists a constant $C>0$ independent of $u$ such that

$$
\left\|v_{j}\right\|_{\mathcal{B}_{\alpha}(\sigma)} \leq C\|u\|_{\mathcal{B}_{\alpha}(\sigma)}
$$

First, we show that the $(n+1)$-tuple $\left(v_{1}, \ldots, v_{n}, u\right)$ satisfies $(1.1),(1.2)$, and (1.3). By (3) of Lemma 3.3, we give

$$
\partial_{k} v_{j}(x, t)=-\frac{2^{\sigma+2}}{\Gamma(\sigma+2)} \int_{H} s^{\sigma+1} \mathcal{D}_{t} u(y, s) \partial_{k} \partial_{j} \mathcal{D}_{t}^{\sigma+1-(1 / 2 \alpha)} W^{(\alpha)}(x-y, t+s) d V(y, s) .
$$

Thus, $\left(v_{1}, \ldots, v_{n}, u\right)$ satisfies (1.1). We show that (1.2) is satisfied. In fact, Lemma 3.2 implies that the function $u$ satisfies

$$
u(x, t)=\frac{2^{\sigma+2}}{\Gamma(\sigma+2)} \Pi_{\alpha}^{0, \sigma+1,0}\left(s^{\sigma+1} \mathcal{D}_{t} u\right)(x, t) .
$$

Hence, (2) and (3) of Lemma 3.3 give 


$$
-\mathcal{D}_{t}^{1 / 2 \alpha} v_{j}(x, t)=\frac{2^{\sigma+2}}{\Gamma(\sigma+2)} \int_{H} s^{\sigma+1} \mathcal{D}_{t} u(y, s) \partial_{j} \mathcal{D}_{t}^{\sigma+1} W^{(\alpha)}(x-y, t+s) d V(y, s)
$$

and

$$
\partial_{j} u(x, t)=\frac{2^{\sigma+2}}{\Gamma(\sigma+2)} \int_{H} s^{\sigma+1} \mathcal{D}_{t} u(y, s) \partial_{j} \mathcal{D}_{t}^{\sigma+1} W^{(\alpha)}(x-y, t+s) d V(y, s) .
$$

Therefore, $\left(v_{1}, \ldots, v_{n}, u\right)$ satisfies (1.2). We show that (1.3) is satisfied. By (4.2), we obtain

$$
\begin{aligned}
& \sum_{j=1}^{n} \partial_{j} v_{j}(x, t) \\
& =\frac{2^{\sigma+2}}{\Gamma(\sigma+2)} \int_{H} s^{\sigma+1} \mathcal{D}_{t} u(y, s) \mathcal{D}_{t}^{\sigma+1-(1 / 2 \alpha)}\left(-\Delta_{x} W^{(\alpha)}(x-y, t+s)\right) d V(y, s) .
\end{aligned}
$$

Here, (2) and (4) of Lemma 2.1 show that

$$
\mathcal{D}_{t}^{\sigma+1-(1 / 2 \alpha)}\left(-\Delta_{x} W^{(\alpha)}(x-y, t+s)\right)=\mathcal{D}_{t}^{\sigma+1+(1 / 2 \alpha)} W^{(\alpha)}(x-y, t+s) .
$$

Consequently, we obtain

$$
\sum_{j=1}^{n} \partial_{j} v_{j}(x, t)=\frac{2^{\sigma+2}}{\Gamma(\sigma+2)} \int_{H} s^{\sigma+1} \mathcal{D}_{t} u(y, s) \mathcal{D}_{t}^{\sigma+1+(1 / 2 \alpha)} W^{(\alpha)}(x-y, t+s) d V(y, s) .
$$

On the other hand, (2) of Lemma 3.3 gives

$$
\mathcal{D}_{t}^{1 / 2 \alpha} u(x, t)=\frac{2^{\sigma+2}}{\Gamma(\sigma+2)} \int_{H} s^{\sigma+1} \mathcal{D}_{t} u(y, s) \mathcal{D}_{t}^{\sigma+1+(1 / 2 \alpha)} W^{(\alpha)}(x-y, t+s) d V(y, s) .
$$

Hence, $\left(v_{1}, \ldots, v_{n}, u\right)$ satisfies $(1.3)$.

Next, we show that the $(n+1)$-tuple $\left(v_{1}, \ldots, v_{n}, u\right)$ satisfies the inequalities (1.4). Since the second inequality is already given by (4.1), it suffices to show the first inequality. By Lemma 3.4 and (1.3), there exists a constant $C>0$ independent of $u$ such that

$$
\|u\|_{\mathcal{B}_{\alpha}(\sigma)} \leq C\left\|t^{(1 / 2 \alpha)+\sigma} \mathcal{D}_{t}^{1 / 2 \alpha} u\right\|_{L^{\infty}} \leq C \sum_{j=1}^{n}\left\|t^{(1 / 2 \alpha)+\sigma} \partial_{j} v_{j}\right\|_{L^{\infty}}
$$

Since $\left\|t^{(1 / 2 \alpha)+\sigma} \partial_{j} v_{j}\right\|_{L^{\infty}} \leq\left\|v_{j}\right\|_{\mathcal{B}_{\alpha}(\sigma)}$, we obtain the first inequality.

Finally, we show the uniqueness of $\left(v_{1}, \ldots, v_{n}\right)$. Suppose that $\left(u_{1}, \ldots, u_{n}\right)$ is an $L^{(\alpha)}$-conjugate of $u$ with $u_{j} \in \widetilde{\mathcal{B}}_{\alpha}(\sigma)$. We show that $v_{j}=u_{j}$ on $H$. By Lemma 3.4 and (1.2), there exists a constant $C>0$ independent of $v_{j}$ and $u_{j}$ such that

$$
\left\|v_{j}-u_{j}\right\|_{\mathcal{B}_{\alpha}(\sigma)} \leq C\left\|t^{(1 / 2 \alpha)+\sigma} \mathcal{D}_{t}^{1 / 2 \alpha}\left(v_{j}-u_{j}\right)\right\|_{L^{\infty}} \leq C\left\|t^{(1 / 2 \alpha)+\sigma}\left(\partial_{j} u-\partial_{j} u\right)\right\|_{L^{\infty}}=0 .
$$

Therefore, we obtain $v_{j}=u_{j}$ on $H$. This completes the proof.

Next, we give the proof of Theorem 2 . 
Proof of Theorem 2. Suppose an $n$-tuple $\left(v_{1}, \ldots, v_{n}\right)$ satisfies $v_{j} \in \widetilde{\mathcal{B}}_{\alpha}(\sigma)$ and (1.1). We define a function $u$ on $H$ by

$$
u(x, t)=\frac{2^{\sigma+2}}{\Gamma(\sigma+2)} \sum_{j=1}^{n} \Pi_{\alpha}^{\gamma(j), \sigma+1-(1 / 2 \alpha), 0}\left(s^{\sigma+1} \mathcal{D}_{t} v_{j}\right)(x, t) .
$$

Since $s^{\sigma+1} \mathcal{D}_{t} v_{j} \in L^{\infty}(H),(1)$ of Lemma 3.3 implies that $u \in \widetilde{\mathcal{B}}_{\alpha}(\sigma)$ and there exists a constant $C>0$ independent of $v_{j}$ such that

$$
\|u\|_{\mathcal{B}_{\alpha}(\sigma)} \leq C \sum_{j=1}^{n}\left\|v_{j}\right\|_{\mathcal{B}_{\alpha}(\sigma)}
$$

First, we show that the $(n+1)$-tuple $\left(v_{1}, \ldots, v_{n}, u\right)$ satisfies (1.2) and (1.3). In fact, Lemma 3.2 implies that the function $v_{j}$ satisfies

$$
v_{j}(x, t)=\frac{2^{\sigma+2}}{\Gamma(\sigma+2)} \Pi_{\alpha}^{0, \sigma+1,0}\left(s^{\sigma+1} \mathcal{D}_{t} v_{j}\right)(x, t) .
$$

Hence, (3) of Lemma 3.3 gives

$$
\begin{aligned}
& \mathcal{D}_{t}^{-1 / 2 \alpha} \partial_{j} \partial_{k} v_{j}(x, t) \\
& =\frac{2^{\sigma+2}}{\Gamma(\sigma+2)} \int_{H} s^{\sigma+1} \mathcal{D}_{t} v_{j}(y, s) \partial_{k} \partial_{j} \mathcal{D}_{t}^{\sigma+1-(1 / 2 \alpha)} W^{(\alpha)}(x-y, t+s) d V(y, s) .
\end{aligned}
$$

On the other hand, (3) of Lemma 3.3 implies that

$$
\partial_{k} u(x, t)=\frac{2^{\sigma+2}}{\Gamma(\sigma+2)} \sum_{j=1}^{n} \int_{H} s^{\sigma+1} \mathcal{D}_{t} v_{j}(y, s) \partial_{k} \partial_{j} \mathcal{D}_{t}^{\sigma+1-(1 / 2 \alpha)} W^{(\alpha)}(x-y, t+s) d V(y, s) .
$$

Therefore, we obtain $\partial_{k} u(x, t)=\sum_{j=1}^{n} \mathcal{D}_{t}^{-1 / 2 \alpha} \partial_{j} \partial_{k} v_{j}(x, t)$. Since $\left(v_{1}, \ldots, v_{n}\right)$ satisfies (1.1), (2) and (4) of Lemma 3.1 give

$$
\partial_{k} u(x, t)=\sum_{j=1}^{n} \mathcal{D}_{t}^{-1 / 2 \alpha} \partial_{j}^{2} v_{k}(x, t)=-\mathcal{D}_{t}^{-1 / 2 \alpha}\left(-\Delta_{x} v_{k}(x, t)\right)=-\mathcal{D}_{t}^{1 / 2 \alpha} v_{k}(x, t) .
$$

Therefore, $\left(v_{1}, \ldots, v_{n}, u\right)$ satisfies (1.2). We show that (1.3) is satisfied. By (2) and (3) of Lemma 3.3, we obtain

$$
\mathcal{D}_{t}^{1 / 2 \alpha} u(x, t)=\frac{2^{\sigma+2}}{\Gamma(\sigma+2)} \sum_{j=1}^{n} \int_{H} s^{\sigma+1} \mathcal{D}_{t} v_{j}(y, s) \partial_{j} \mathcal{D}_{t}^{\sigma+1} W^{(\alpha)}(x-y, t+s) d V(y, s)
$$

and

$$
\partial_{j} v_{j}(x, t)=\frac{2^{\sigma+2}}{\Gamma(\sigma+2)} \int_{H} s^{\sigma+1} \mathcal{D}_{t} v_{j}(y, s) \partial_{j} \mathcal{D}_{t}^{\sigma+1} W^{(\alpha)}(x-y, t+s) d V(y, s) .
$$


Hence, $\left(v_{1}, \ldots, v_{n}, u\right)$ satisfies (1.3).

Next, we show that the $(n+1)$-tuple $\left(v_{1}, \ldots, v_{n}, u\right)$ satisfies the inequalities (1.5). Since the second inequality is already given by (4.4), it suffices to show the first inequality. By Lemma 3.4 and (1.2), there exists a constant $C>0$ independent of $v_{j}$ such that

$$
\left\|v_{j}\right\|_{\mathcal{B}_{\alpha}(\sigma)} \leq C\left\|t^{(1 / 2 \alpha)+\sigma} \mathcal{D}_{t}^{1 / 2 \alpha} v_{j}\right\|_{L^{\infty}}=C\left\|t^{(1 / 2 \alpha)+\sigma} \partial_{j} u\right\|_{L^{\infty}} .
$$

Since $\left\|t^{(1 / 2 \alpha)+\sigma} \partial_{j} u\right\|_{L^{\infty}} \leq\|u\|_{\mathcal{B}_{\alpha}(\sigma)}$, we obtain the first inequality.

Finally, we show the uniqueness of the function $u$. Suppose that $v \in \widetilde{\mathcal{B}}_{\alpha}(\sigma)$ and the $n$-tuple $\left(v_{1}, \ldots, v_{n}\right)$ is an $L^{(\alpha)}$-conjugate of $v$. We show that $u=v$ on $H$. By Lemma 3.4 and (1.3), there exists a constant $C>0$ independent of $u$ and $v$ such that

$$
\begin{aligned}
\|u-v\|_{\mathcal{B}_{\alpha}(\sigma)} & \leq C\left\|t^{(1 / 2 \alpha)+\sigma}\left(\mathcal{D}_{t}^{1 / 2 \alpha} u-\mathcal{D}_{t}^{1 / 2 \alpha} v\right)\right\|_{L^{\infty}} \\
& =C\left\|t^{(1 / 2 \alpha)+\sigma}\left(\sum_{j=1}^{n} \partial_{j} v_{j}-\sum_{j=1}^{n} \partial_{j} v_{j}\right)\right\|_{L^{\infty}}=0 .
\end{aligned}
$$

Therefore, we obtain $u=v$ on $H$. This completes the proof.

\section{A generalization of $L^{(\alpha)}$-conjugates.}

In this section, we discuss a generalization of $L^{(\alpha)}$-conjugates. We generalize the definition of $L^{(\alpha)}$-conjugates, and study properties of generalized $L^{(\alpha)}$-conjugates. We introduce the following definition, which is called a $(\kappa, \nu)$-conjugate.

Definition 5.1. Let $u$ be a function on $H$. An $n$-tuple $\left(v_{1}, \ldots, v_{n}\right)$ is called a $(\kappa, \nu)$-conjugate of $u$ if $u, v_{j} \in C^{1}(H), v_{j}(x, \cdot) \in \mathcal{F C}^{\kappa}, u(x, \cdot) \in \mathcal{F} \mathcal{C}^{\nu}$, and the $(n+1)$-tuple $\left(v_{1}, \ldots, v_{n}, u\right)$ satisfies the following equations:

$$
\begin{aligned}
& \partial_{j} v_{k}=\partial_{k} v_{j}(1 \leq j, k \leq n), \\
& -\mathcal{D}_{t}^{\kappa} v_{j}=\left\{\begin{array}{ll}
\partial_{j} u & (\kappa \neq 0) \\
\partial_{j} u-\partial_{j} u(0,1) & (\kappa=0)
\end{array}(1 \leq j \leq n),\right.
\end{aligned}
$$

and

$$
\sum_{j=1}^{n} \partial_{j} v_{j}= \begin{cases}\mathcal{D}_{t}^{\nu} u & (\nu \neq 0) \\ u-\lim _{t \rightarrow \infty} u(0, t) & (\nu=0) .\end{cases}
$$

We note that $(1 / 2 \alpha, 1 / 2 \alpha)$-conjugates and $(1,(1 / \alpha)-1)$-conjugates coincide with $L^{(\alpha)}$-conjugates and $\alpha$-parabolic conjugate functions, respectively. Therefore, the study of $(\kappa, \nu)$-conjugates is an extension of Section 4 and the previous paper [4].

Now, we start investigation of $(\kappa, \nu)$-conjugates on parabolic Bloch spaces. In order to show the main theorem of this section, we prepare the following lemmas.

Lemma 5.2. Let $0<\alpha \leq 1, \sigma>-m(\alpha)$, and $u \in \mathcal{B}_{\alpha}(\sigma)$. Also, let $\gamma \in \mathbb{N}_{0}^{n}$, $\kappa>\max \{0,-\sigma\}$, and $\nu>\sigma$. If $\sigma>-|\gamma| / 2 \alpha$, then 


$$
\int_{H}\left|\mathcal{D}_{t}^{\kappa} u(y, s)\right|\left|\partial_{x}^{\gamma} \mathcal{D}_{t}^{\nu} W^{(\alpha)}(x-y, t+s)\right| s^{\kappa+\nu-1} d V(y, s)<\infty
$$

for all $(x, t) \in H$.

Proof. The proof is given by (1) of Lemma 2.1 and Lemma 2.3, immediately.

Lemma 5.3 (Lemma 5.2 of [4]). Let $0<\alpha \leq 1, \sigma>0, u \in \widetilde{\mathcal{B}}_{\alpha}(\sigma)$, and $(x, t) \in H$. If $\kappa>0$ and $\nu>\sigma$, then

$$
\begin{aligned}
& u(x, t)-\lim _{s \rightarrow \infty} u(x . s) \\
& =\frac{2^{\kappa+\nu}}{\Gamma(\kappa+\nu)} \int_{H} \mathcal{D}_{t}^{\kappa} u(y, s) \mathcal{D}_{t}^{\nu} W^{(\alpha)}(x-y, t+s) s^{\kappa+\nu-1} d V(y, s) .
\end{aligned}
$$

As an application of $(\kappa, \nu)$-conjugates, we can generalize Theorem 1.

TheOrem 5.4. Let $0<\alpha \leq 1, \sigma>-m(\alpha)$, and $u \in \widetilde{\mathcal{B}}_{\alpha}(\sigma)$. Also, let $\kappa$ and $\nu$ be non-negative real numbers with $\kappa+\nu=1 / \alpha$. Put $\eta=(1 / 2 \alpha)+\sigma-\kappa$. If $\eta>-m(\alpha)$, then there exists a unique $(\kappa, \nu)$-conjugate $\left(v_{1}, \cdots, v_{n}\right)$ of $u$ such that $v_{j} \in \widetilde{\mathcal{B}}_{\alpha}(\eta)$. Moreover, there exists a constant $C>0$ independent of $u$ such that

$$
C^{-1}\|u\|_{\mathcal{B}_{\alpha}(\sigma)} \leq \sum_{j=1}^{n}\left\|v_{j}\right\|_{\mathcal{B}_{\alpha}(\eta)} \leq C\|u\|_{\mathcal{B}_{\alpha}(\sigma)}
$$

Proof. Let $u \in \widetilde{\mathcal{B}}_{\alpha}(\sigma)$. For $1 \leq j \leq n$, we define a function $v_{j}$ on $H$ by

$$
v_{j}(x, t)=-\frac{2^{\sigma+2}}{\Gamma(\sigma+2)} \Pi_{\alpha}^{\gamma(j), \sigma+1-\kappa, 0}\left(s^{\sigma+1} \mathcal{D}_{t} u\right)(x, t) .
$$

Since $s^{\sigma+1} \mathcal{D}_{t} u \in L^{\infty}(H)$, (1) of Lemma 3.3 implies that $v_{j} \in \widetilde{\mathcal{B}}_{\alpha}(\eta)$ and there exists a constant $C>0$ independent of $u$ such that

$$
\left\|v_{j}\right\|_{\mathcal{B}_{\alpha}(\eta)} \leq C\|u\|_{\mathcal{B}_{\alpha}(\sigma)} .
$$

We show that the $(n+1)$-tuple $\left(v_{1}, \ldots, v_{n}, u\right)$ satisfies (5.1), (5.2), and (5.3). By (3) of Lemma 3.3, we obtain

$$
\partial_{k} v_{j}(x, t)=\frac{2^{\sigma+2}}{\Gamma(\sigma+2)} \int_{H} s^{\sigma+1} \mathcal{D}_{t} u(y, s) \partial_{k} \partial_{j} \mathcal{D}_{t}^{\sigma+1-\kappa} W^{(\alpha)}(x-y, t+s) d V(y, s) .
$$

Hence, $\left(v_{1}, \ldots, v_{n}, u\right)$ satisfies (5.1). We show that (5.2) is satisfied. In fact, Lemma 3.2 implies that the function $u$ satisfies

$$
u(x, t)=\frac{2^{\sigma+2}}{\Gamma(\sigma+2)} \Pi_{\alpha}^{0, \sigma+1,0}\left(s^{\sigma+1} \mathcal{D}_{t} u\right)(x, t) .
$$

Therefore, (3) of Lemma 3.3 shows that 


$$
\partial_{j} u(x, t)=\frac{2^{\sigma+2}}{\Gamma(\sigma+2)} \int_{H} s^{\sigma+1} \mathcal{D}_{t} u(y, s) \partial_{j} \mathcal{D}_{t}^{\sigma+1} W^{(\alpha)}(x-y, t+s) d V(y, s) .
$$

Let $\kappa>0$. Since $\kappa>-\eta$, (2) of Lemma 3.3 implies that

$$
-\mathcal{D}_{t}^{\kappa} v_{j}(x, t)=\frac{2^{\sigma+2}}{\Gamma(\sigma+2)} \int_{H} s^{\sigma+1} \mathcal{D}_{t} u(y, s) \partial_{j} \mathcal{D}_{t}^{\sigma+1} W^{(\alpha)}(x-y, t+s) d V(y, s) .
$$

Therefore, (5.2) is satisfied when $\kappa>0$. Let $\kappa=0$. By Lemma 5.2 and (5.8), we obtain

$$
\begin{aligned}
-v_{j}(x, t)= & \frac{2^{\sigma+2}}{\Gamma(\sigma+2)} \int_{H} s^{\sigma+1} \mathcal{D}_{t} u(y, s) \partial_{j} \mathcal{D}_{t}^{\sigma+1} W^{(\alpha)}(x-y, t+s) d V(y, s) \\
& -\frac{2^{\sigma+2}}{\Gamma(\sigma+2)} \int_{H} s^{\sigma+1} \mathcal{D}_{t} u(y, s) \partial_{j} \mathcal{D}_{t}^{\sigma+1} W^{(\alpha)}(-y, 1+s) d V(y, s) \\
= & \partial_{j} u(x, t)-\partial_{j} u(0,1) .
\end{aligned}
$$

Consequently, (5.2) is satisfied for $\kappa \geq 0$. We show that (5.3) is satisfied. By (3) of Lemma 3.3, we have

$$
\sum_{j=1}^{n} \partial_{j} v_{j}(x, t)=\frac{2^{\sigma+2}}{\Gamma(\sigma+2)} \int_{H} s^{\sigma+1} \mathcal{D}_{t} u(y, s) \mathcal{D}_{t}^{\sigma+1-\kappa}\left(-\Delta_{x} W^{(\alpha)}(x-y, t+s)\right) d V(y, s) .
$$

Here, (2) and (4) of Lemma 2.1 give

$$
\mathcal{D}_{t}^{\sigma+1-\kappa}\left(-\Delta_{x} W^{(\alpha)}(x-y, t+s)\right)=\mathcal{D}_{t}^{\sigma+1+\nu} W^{(\alpha)}(x-y, t+s) .
$$

Therefore, we obtain

$$
\sum_{j=1}^{n} \partial_{j} v_{j}(x, t)=\frac{2^{\sigma+2}}{\Gamma(\sigma+2)} \int_{H} s^{\sigma+1} \mathcal{D}_{t} u(y, s) \mathcal{D}_{t}^{\sigma+1+\nu} W^{(\alpha)}(x-y, t+s) d V(y, s) .
$$

Let $\nu>0$. Then, it follows from (2) of Lemma 3.3 that

$$
\mathcal{D}_{t}^{\nu} u(x, t)=\frac{2^{\sigma+2}}{\Gamma(\sigma+2)} \int_{H} s^{\sigma+1} \mathcal{D}_{t} u(y, s) \mathcal{D}_{t}^{\sigma+1+\nu} W^{(\alpha)}(x-y, t+s) d V(y, s) .
$$

Therefore, (5.3) is satisfied when $\nu>0$. Let $\nu=0$. Then, Lemmas 5.2 and 5.3 imply that

$$
\begin{aligned}
u(x, t)= & \frac{2^{\sigma+2}}{\Gamma(\sigma+2)} \int_{H} s^{\sigma+1} \mathcal{D}_{t} u(y, s) \omega_{\alpha}^{0, \sigma+1}(x, t ; y, s) d V(y, s) \\
= & \frac{2^{\sigma+2}}{\Gamma(\sigma+2)} \int_{H} s^{\sigma+1} \mathcal{D}_{t} u(y, s) \mathcal{D}_{t}^{\sigma+1} W^{(\alpha)}(x-y, t+s) d V(y, s) \\
& -\frac{2^{\sigma+2}}{\Gamma(\sigma+2)} \int_{H} s^{\sigma+1} \mathcal{D}_{t} u(y, s) \mathcal{D}_{t}^{\sigma+1} W^{(\alpha)}(-y, 1+s) d V(y, s) \\
= & \sum_{j=1}^{n} \partial_{j} v_{j}(x, t)-\left\{u(0,1)-\lim _{s \rightarrow \infty} u(0, s)\right\}=\sum_{j=1}^{n} \partial_{j} v_{j}(x, t)+\lim _{s \rightarrow \infty} u(0, s) .
\end{aligned}
$$


Thus, (5.3) is satisfied for $\nu \geq 0$.

Next, we show the inequalities (5.4). The second inequality is already given by (5.5). Thus, it suffices to show the first inequality. If $\nu>0$, then Lemma 3.4 and (5.3) shows that there exists a constant $C>0$ independent of $u$ such that

$$
\|u\|_{\mathcal{B}_{\alpha}(\sigma)} \leq C\left\|t^{\sigma+\nu} \mathcal{D}_{t}^{\nu} u\right\|_{L^{\infty}}=C\left\|t^{\sigma+\nu} \sum_{j=1}^{n} \partial_{j} v_{j}\right\|_{L^{\infty}} \leq C \sum_{j=1}^{n}\left\|t^{\sigma+\nu} \partial_{j} v_{j}\right\|_{L^{\infty}} .
$$

Since $\left|\partial_{j} v_{j}\right| \leq\left|\nabla_{x} v_{j}\right|$ and $\sigma+\nu=\eta+(1 / 2 \alpha)$, we obtain

$$
\|u\|_{\mathcal{B}_{\alpha}(\sigma)} \leq C \sum_{j=1}^{n}\left\|t^{\eta} t^{1 / 2 \alpha} \nabla_{x} v_{j}\right\|_{L^{\infty}} \leq C \sum_{j=1}^{n}\left\|v_{j}\right\|_{\mathcal{B}_{\alpha}(\eta)} .
$$

We assume $\nu=0$. Since the limit value $\lim _{s \rightarrow \infty} u(0, s)$ exists (see, Remark 5.6 of $[4]$ ), (5.3) gives

$$
\|u\|_{\mathcal{B}_{\alpha}(\sigma)}=\left\|\sum_{j=1}^{n} \partial_{j} v_{j}\right\|_{\mathcal{B}_{\alpha}(\sigma)}
$$

Also, (1) of Lemma 3.1 implies that there exists a constant $C>0$ independent of $v_{j}$ such that

$$
t^{\sigma+(1 / 2 \alpha)}\left|\nabla_{x} \partial_{j} v_{j}(x, t)\right| \leq C t^{\sigma-(1 / 2 \alpha)-\eta}\left\|v_{j}\right\|_{\mathcal{B}_{\alpha}(\eta)}=C\left\|v_{j}\right\|_{\mathcal{B}_{\alpha}(\eta)}
$$

and

$$
t^{\sigma+1}\left|\mathcal{D}_{t} \partial_{j} v_{j}(x, t)\right| \leq C t^{\sigma-(1 / 2 \alpha)-\eta}\left\|v_{j}\right\|_{\mathcal{B}_{\alpha}(\eta)}=C\left\|v_{j}\right\|_{\mathcal{B}_{\alpha}(\eta)} .
$$

Therefore, we obtain the first inequality for $\nu \geq 0$.

Finally, we show the uniqueness of the $(\kappa, \nu)$-conjugate $\left(v_{1}, \cdots, v_{n}\right)$ of $u$. Suppose that $\left(u_{1}, \cdots, u_{n}\right)$ is a $(\kappa, \nu)$-conjugate of $u$ such that $u_{j} \in \widetilde{\mathcal{B}}_{\alpha}(\eta)$. We show that $u_{j}=v_{j}$ on $H$. Let $\kappa>0$. Then, by Lemma 3.4 and (5.2), there exists a constant $C>0$ independent of $u$ such that

$$
\left\|u_{j}-v_{j}\right\|_{\mathcal{B}_{\alpha}(\eta)} \leq C\left\|t^{\eta+\kappa}\left(\mathcal{D}_{t}^{\kappa} u_{j}-\mathcal{D}_{t}^{\kappa} v_{j}\right)\right\|_{L^{\infty}}=C\left\|t^{\eta+\kappa}\left(\partial_{j} u-\partial_{j} u\right)\right\|_{L^{\infty}}=0 .
$$

The case of $\kappa=0$ is similar to that of $\kappa>0$. Thus, this completes the proof.

\section{Isomorphism theorem on parabolic Bloch spaces.}

In this section, we shall prove Theorem 3. The study of parabolic Bloch spaces is closely related to that of parabolic Bergman spaces. In fact, parabolic Bloch spaces are regarded as a limiting case $p \rightarrow \infty$ of parabolic Bergman spaces. Thus, we recall the definition of parabolic Bergman spaces. Let $0<\alpha \leq 1,1 \leq p<\infty$, and $\lambda>-1$. The parabolic Bergman space $\boldsymbol{b}_{\alpha}^{p}(\lambda)$ is the space of all $L^{(\alpha)}$-harmonic functions which belong to $L^{p}(\lambda)$, where $L^{p}(\lambda)=L^{p}\left(H, t^{\lambda} d V\right)$ is the set of all Lebesgue measurable functions on 
$H$ which satisfy

$$
\|f\|_{L^{p}(\lambda)}:=\left(\int_{H}|f(x, t)|^{p} t^{\lambda} d V(x, t)\right)^{1 / p}<\infty .
$$

The following proposition is given in the previous paper [5], which is a corresponding result of Theorem 3 .

Theorem B (Theorem 5.4 and Remark 5.5 of [5]). Let $0<\alpha \leq 1,1 \leq p<\infty$, and $\lambda_{1}, \lambda_{2}>-1$. Then, $\boldsymbol{b}_{\alpha}^{p}\left(\lambda_{1}\right) \cong \boldsymbol{b}_{\alpha}^{p}\left(\lambda_{2}\right)$ under the relation $\mathcal{D}_{t}^{-\lambda_{1} / p} u=\mathcal{D}_{t}^{-\lambda_{2} / p} v$ for $u \in \boldsymbol{b}_{\alpha}^{p}\left(\lambda_{1}\right)$ and $v \in \boldsymbol{b}_{\alpha}^{p}\left(\lambda_{2}\right)$. Moreover, there exists a constant $C>0$ such that

$$
C^{-1}\|v\|_{L^{p}\left(\lambda_{2}\right)} \leq\|u\|_{L^{p}\left(\lambda_{1}\right)} \leq C\|v\|_{L^{p}\left(\lambda_{2}\right)} .
$$

Now, we start the investigation of this section. As an application of Theorems 2 and 5.4 , we can give the following theorem.

Theorem 6.1. Let $0<\alpha \leq 1$ and $\sigma_{1}, \sigma_{2}>-m(\alpha)$ be real numbers. Then, $\widetilde{\mathcal{B}}_{\alpha}\left(\sigma_{1}\right) \cong \widetilde{\mathcal{B}}_{\alpha}\left(\sigma_{2}\right)$.

Proof. Without loss of generality, we may assume that $\sigma_{1} \leq \sigma_{2}$. First, we suppose that $\sigma_{2}-\sigma_{1} \leq 1 / 2 \alpha$. Let $u \in \widetilde{\mathcal{B}}_{\alpha}\left(\sigma_{1}\right)$. Then, by Theorem 5.4 with $\kappa=$ $(1 / 2 \alpha)-\sigma_{2}+\sigma_{1}$ and $\nu=(1 / 2 \alpha)+\sigma_{2}-\sigma_{1}$, there exists a unique $(\kappa, \nu)$-conjugate $\left(v_{1}, \ldots, v_{n}\right)$ of $u$ such that $v_{j} \in \widetilde{\mathcal{B}}_{\alpha}\left(\sigma_{2}\right)$. Moreover, by Theorem 2, there exists a unique function $v \in \widetilde{\mathcal{B}}_{\alpha}\left(\sigma_{2}\right)$ such that $\left(v_{1}, \ldots, v_{n}\right)$ is the $L^{(\alpha)}$-conjugate of $v$. Also, Theorems 2 and 5.4 show that there exists a constant $C>0$ independent of $u$ such that

$$
C^{-1}\|u\|_{\mathcal{B}_{\alpha}\left(\sigma_{1}\right)} \leq\|v\|_{\mathcal{B}_{\alpha}\left(\sigma_{2}\right)} \leq C\|u\|_{\mathcal{B}_{\alpha}\left(\sigma_{1}\right)} .
$$

Therefore, we obtain $\widetilde{\mathcal{B}}_{\alpha}\left(\sigma_{1}\right) \cong \widetilde{\mathcal{B}}_{\alpha}\left(\sigma_{2}\right)$ when $0 \leq \sigma_{2}-\sigma_{1} \leq 1 / 2 \alpha$.

In order to show the assertion for general $\sigma_{1}$ and $\sigma_{2}$ with $\sigma_{1} \leq \sigma_{2}$, it suffices to apply the above result several times. Thus, this completes the proof.

REmark 6.2. In Theorem 6.1, we can also find a correspondence between $u \in$ $\widetilde{\mathcal{B}}_{\alpha}\left(\sigma_{1}\right)$ and $v \in \widetilde{\mathcal{B}}_{\alpha}\left(\sigma_{2}\right)$. Actually, if $\sigma_{1} \leq \sigma_{2}$, then (1.3) and (5.3) give

$$
\mathcal{D}_{t}^{1 / 2 \alpha} v=\mathcal{D}_{t}^{(1 / 2 \alpha)+\sigma_{2}-\sigma_{1}} u .
$$

In order to show the equation in Theorem 3, we prepare the following lemmas.

Lemma 6.3 (Proposition 2.1 of $[\mathbf{2}]$ ). Let $\kappa$ and $\nu$ be positive real numbers. If $\varphi \in \mathcal{F C}^{-\nu-\kappa}$, then $\mathcal{D}_{t}^{-\nu} \mathcal{D}_{t}^{-\kappa} \varphi=\mathcal{D}_{t}^{-\nu-\kappa} \varphi$.

LEMma 6.4. Let $0<\alpha \leq 1, \sigma>-m(\alpha)$, and $u \in \widetilde{\mathcal{B}}_{\alpha}(\sigma)$. Also, let $\kappa, \nu>$ $\max \{0,-\sigma\}$ be real numbers. Then, $\mathcal{D}_{t}^{\nu-\kappa} \mathcal{D}_{t}^{\kappa} u(x, t)=\mathcal{D}_{t}^{\nu} u(x, t)$ for all $(x, t) \in H$.

Proof. We assume that $\nu>\kappa$. Then, the differentiation under the integral sign shows that 


$$
\mathcal{D}_{t}^{\nu-\kappa} \mathcal{D}_{t}^{\kappa} u(x, t)=\mathcal{D}_{t}^{-(\lceil\nu-\kappa\rceil-(\nu-\kappa))} \mathcal{D}_{t}^{-(\lceil\kappa\rceil-\kappa)} \mathcal{D}_{t}^{\lceil\nu-\kappa\rceil+\lceil\kappa\rceil} u(x, t) .
$$

In fact, the derivative $\mathcal{D}_{t}^{\lceil\nu-\kappa\rceil+\lceil\kappa\rceil} u(x, t)$ belongs to $\mathcal{F C}^{-(\lceil\nu-\kappa\rceil+\lceil\kappa\rceil-\nu)}$. Hence, Lemma 6.3 gives

$$
\begin{aligned}
& \mathcal{D}_{t}^{-(\lceil\nu-\kappa\rceil-(\nu-\kappa))} \mathcal{D}_{t}^{-(\lceil\kappa\rceil-\kappa)} \mathcal{D}_{t}^{\lceil\nu-\kappa\rceil+\lceil\kappa\rceil} u(x, t) \\
& =\mathcal{D}_{t}^{-(\lceil\nu-\kappa\rceil+\lceil\kappa\rceil-\nu)} \mathcal{D}_{t}^{\lceil\nu-\kappa\rceil+\lceil\kappa\rceil} u(x, t) .
\end{aligned}
$$

If $\lceil\nu-\kappa\rceil+\lceil\kappa\rceil=\lceil\nu\rceil$, then the right-hand side of (6.3) becomes $\mathcal{D}_{t}^{\nu} u(x, t)$, directly. If $\lceil\nu-\kappa\rceil+\lceil\kappa\rceil=\lceil\nu\rceil+1$, then the right-hand side of (6.3) becomes

$$
\mathcal{D}_{t}^{-(\lceil\nu\rceil-\nu)-1} \mathcal{D}_{t}^{\lceil\nu\rceil+1} u(x, t)=\mathcal{D}_{t}^{-(\lceil\nu\rceil-\nu)} \mathcal{D}_{t}^{-1} \mathcal{D}_{t} \mathcal{D}_{t}^{\lceil\nu\rceil} u(x, t) .
$$

Therefore, (1) of Lemma 3.1 implies that the right-hand side of (6.4) is equal to $\mathcal{D}_{t}^{\nu} u(x, t)$.

We assume that $\nu<\kappa$. Since $\nu>-\sigma$, Lemma 6.3 shows that

$$
\begin{aligned}
\mathcal{D}_{t}^{\nu-\kappa} \mathcal{D}_{t}^{\kappa} u(x, t) & =\mathcal{D}_{t}^{\nu-\lceil\kappa\rceil} \mathcal{D}_{t}^{\lceil\kappa\rceil} u(x, t) \\
& =\mathcal{D}_{t}^{-(\lceil\nu\rceil-\nu)} \mathcal{D}_{t}^{-\lceil\kappa\rceil+\lceil\nu\rceil} \mathcal{D}_{t}^{\lceil\kappa\rceil-\lceil\nu\rceil} \mathcal{D}_{t}^{\lceil\nu\rceil} u(x, t)
\end{aligned}
$$

Therefore, (1) of Lemma 3.1 implies that the right-hand side of $(6.5)$ is equal to $\mathcal{D}_{t}^{\nu} u(x, t)$. This completes the proof.

Now, we give the proof of Theorem 3 .

Proof of Theorem 3. Without loss of generality, we may assume that $\sigma_{1} \leq \sigma_{2}$. Then, Theorem 6.1 and (6.2) imply that $\widetilde{\mathcal{B}}_{\alpha}\left(\sigma_{1}\right) \cong \widetilde{\mathcal{B}}_{\alpha}\left(\sigma_{2}\right)$ under the correspondence $\mathcal{D}_{t}^{1 / 2 \alpha} v=\mathcal{D}_{t}^{(1 / 2 \alpha)+\sigma_{2}-\sigma_{1}} u$ for $u \in \widetilde{\mathcal{B}}_{\alpha}\left(\sigma_{1}\right)$ and $v \in \widetilde{\mathcal{B}}_{\alpha}\left(\sigma_{2}\right)$. Since $\kappa>\max \left\{0, \sigma_{1}, \sigma_{2}\right\}$, Lemma 6.4 gives

$$
\mathcal{D}_{t}^{\kappa-\sigma_{2}-(1 / 2 \alpha)} \mathcal{D}_{t}^{(1 / 2 \alpha)+\sigma_{2}-\sigma_{1}} u=\mathcal{D}_{t}^{-\sigma_{1}+\kappa} u
$$

and

$$
\mathcal{D}_{t}^{\kappa-\sigma_{2}-(1 / 2 \alpha)} \mathcal{D}_{t}^{1 / 2 \alpha} v=\mathcal{D}_{t}^{-\sigma_{2}+\kappa} v .
$$

Consequently, we obtain $\mathcal{D}_{t}^{-\sigma_{1}+\kappa} u=\mathcal{D}_{t}^{-\sigma_{2}+\kappa} v$.

The inequalities (1.6) are given by (6.1), directly. Thus, this completes the proof.

\section{References}

[1] E. A. Abdel-Rehim, Fundamental solutions of the fractional diffusion and the fractional FokkerPlanck equations, J. Egyptian Math. Soc., 24 (2016), 337-347.

[2] Y. Hishikawa, Fractional calculus on parabolic Bergman spaces, Hiroshima Math. J., 38 (2008), 471-488.

[ 3 ] Y. Hishikawa, M. Nishio and M. Yamada, A conjugate system and tangential derivative norms on parabolic Bergman spaces, Hokkaido Math. J., 39 (2010), 85-114.

[4] Y. Hishikawa, M. Nishio and M. Yamada, Conjugate functions on spaces of parabolic Bloch type, J. Math. Soc. Japan, 65 (2013), 487-520. 
[ 5 ] Y. Hishikawa, M. Nishio and M. Yamada, $L^{(\alpha)}$-conjugates on parabolic Bergman spaces, Potential Anal., 40 (2014), 525-537.

[6] Y. Hishikawa and M. Yamada, Function spaces of parabolic Bloch type, Hiroshima Math. J., 41 (2011), 55-87.

[ 7 ] M. Itô and M. Nishio, Poincaré type conditions of the regularity for the parabolic operator of order $\alpha$, Nagoya Math. J., 115 (1989), 1-22.

[ 8 ] J. Jia and K. Li, Maximal principles for a time-space fractional diffusion equation, Appl. Math. Lett., 62 (2016), 23-28.

[ 9 ] R. Jiang, J. Xiao, D. Yang and Z. Zhai, Regularity and capacity for the fractional dissipative operator, J. Differential Equations, 259 (2015), 3495-3519.

[10] E. Kochneff and Y. Sagher, Conjugate temperatures, J. Approx. Theory, 70 (1992), 39-49.

[11] M. Nishio, K. Shimomura and N. Suzuki, $\alpha$-parabolic Bergman spaces, Osaka J. Math., 42 (2005), 133-162.

[12] M. Nishio, N. Suzuki and M. Yamada, Toeplitz operators and Carleson measures on parabolic Bergman spaces, Hokkaido Math. J., 36 (2007), 563-583.

[13] W. Ramey and H. Yi, Harmonic Bergman functions on half-spaces, Trans. Amer. Math. Soc., 348 (1996), 633-660.

[14] E. M. Stein and G. Weiss, On the theory of harmonic functions of several variables I, The theory of $H^{p}$-spaces, Acta Math., 103 (1960), 25-62.

\section{Yôsuke HisHIKAWA}

Department of Mathematics

Faculty of Education

Gifu University

Yanagido 1-1

Gifu 501-1193, Japan

E-mail: yhishik@gifu-u.ac.jp

\author{
Masaharu Nishio \\ Department of Mathematics \\ Osaka City University \\ Sugimoto, Sumiyoshi 3-3-138 \\ Osaka 558-8585, Japan \\ E-mail: nishio@sci.osaka-cu.ac.jp
}

\section{Masahiro YAMADA}

Department of Mathematics

Faculty of Education

Gifu University

Yanagido 1-1

Gifu 501-1193, Japan

E-mail: yamada33@gifu-u.ac.jp 